\title{
Patent Foramen Ovale: Risk Factors and Diagnostic Comparison of Transcranial Doppler versus Echocardiography for Secondary Stroke Prevention
}

Konark Malhotra*, Aristeidis H. Katsanos ${ }^{\dagger,}$, Alexandra Frogoudaki ${ }^{\natural}$, Agathi-Rosa Vrettou ${ }^{\natural}$, Ignatios Ikonomidis ${ }^{\natural}$, Georgios Tsivgoulis ${ }^{\ddagger}, \|$

Department of Neurology, Charleston Area Medical Center, West Virginia University*, Charleston, WV, USA; Department of Neurology, St Josef Hospital, Ruhr University of Bochum ${ }^{\dagger}$, Bochum, Germany; Second Department of Neurology, National \& Kapodistrian University of Athens, "Attikon" University Hospital ${ }^{\ddagger}$, Athens; Second Department of Cardiology, National \& Kapodistrian University of Athens, "Attikon" University Hospital $\left.\right|^{\mid}$, Athens, Greece; Department of Neurology, University of Tennessee Health Science Center", Memphis, TN, USA

Patent foramen ovale (PFO) is the most common cause of right-to-left shunt that increases the potential for paradoxical embolism. Management of patients with cryptogenic ischemic stroke with PFO pose diagnostic and therapeutic challenges for clinicians. In this review article, we briefly discuss various factors associated with PFO that increase its causal relationship with cryptogenic ischemic stroke. We extensively discuss various diagnostic modalities (transcranial Doppler vs. transthoracic or transesophageal echocardiography) available for detection and detailed evaluation of PFO, compare the advantages and disadvantages among these diagnostic modalities and further provide the updated recommendations for medical and interventional treatment options for cryptogenic stroke patients diagnosed with PFO.

J Neurosonol Neuroimag 2019;11(1):22-33

Key Words: Patent foramen ovale; Transcranial doppler ultrasonography; Echocardiography; Cryptogenic ischemic stroke
Received: March 17, 2019

Revised: April 18, 2019

Accepted: May 2, 2019

Address for correspondence: Georgios Tsivgoulis

Second Department of Neurology, University of Athens, School of Medicine, Iras 39, Gerakas Attikis, Athens 15344, Greece Tel: $+30-6937178635$ Fax: $+30-2105832471$ E-mail: tsivgoulisgiorg@yahoo.gr

\section{INTRODUCTION}

Patent foramen ovale ( $\mathrm{PFO}$ ) is prevalent in one-quarter of all adults and is the most common cause of right-to-left shunt (RLS). ${ }^{1}$ Annually, 18,000 patients between 18 to 60 years of age living in the United States and 345,000 worldwide are diagnosed with a PFO and an embolic stroke of otherwise undetermined source (ESUS). ${ }^{2}$ ESUS represents a substantial portion of cryptogenic ischemic strokes that may have paradoxical embolism due to $\mathrm{PFO}$ as an underlying etiology. ${ }^{3} \mathrm{PFO}$ is prevalent in one half of patients with cryptogenic stroke, ${ }^{3}$ and one-half of those PFOs are causative for ischemic strokes. ${ }^{4}$ PFO provides ample conduit for deep vein thrombi (DVT) to bypass downstream pulmonary filtration, and instead paradoxically cross right-to-left cardiac chambers to occlude distal cerebral arteries. As the diagnostic management of cryptogenic stroke remains a challenge for clinicians, detection of PFO followed by detailed evaluation represents a critical part of stroke management.

Multiple randomized-controlled clinical trials (RCTs), earlier trials in 2012 to $2013,{ }^{5-7}$ followed by in 2017 to $2018^{8-11}$ have transformed the diagnostic and therapeutic management for cryptogenic stroke patients diagnosed with PFO. However, various available diagnostic modalities continue to pose clinical dilemma for clinicians during the management of cryptogenic ischemic stroke patients. In this review, we discuss the factors associated with PFO that increase its causal relationship with cryptogenic ischemic stroke, various diagnostic modalities available for PFO detection (transcranial Doppler [TCD] vs. transthoracic or transesophageal echocardiography [TEE]) and compare their technical advantages and disadvantages and provide updated diagnostic and treatment management recommendations 
in cryptogenic stroke patients diagnosed with PFO.

\section{PFO RELATED VARIABLES ASSOCIATED WITH CRYPTOGENIC STROKE}

Various factors correlated to PFO that tend to increase its causal relationship with cryptogenic ischemic stroke include: young age, valsalva maneuver (VM), recent prolonged immobility, concomitant DVT in legs or pelvis, presence of atrial septal aneurysm (ASA), history of migraine with aura, and neuroimaging demonstrating solitary cortical or bilateral embolic pattern. ${ }^{3}$ Few of these factors have been discussed in the following section.

\section{Age}

PFO has a higher prevalence ( $-50-60 \%)$ among young and middle-aged (age, 18-6o years) patients with cryptogenic ischemic stroke. ${ }^{2}$ In comparison to age-matched patients with a known cause of ischemic stroke, prior pooled analysis of case-control studies assessed the prevalence of PFO and its probability of being an incidental finding among patients with cryptogenic stroke. ${ }^{12}$ Using additional estimate correction for potential publication bias, a recent topical review demonstrated that the presence of PFO among young and middle-aged patients with cryptogenic ischemic stroke is associated with a relative increased risk of stroke by 2.3 -fold. ${ }^{2}$ Of all the strokes among young and middle-aged patients harboring a $\mathrm{PFO}, 73 \%$ of strokes are accounted by $\mathrm{PFO}$ using probability theorem whereas rest $27 \%$ are incidental in nature.

\section{Atrial septal aneurysm}

The prevalence of ASA, congenital malformation of the septum primum, ranges from $2-3 \%$ in the general population. ${ }^{.}$The term 'aneurysm' is a misnomer, as it is defined as an excursion of interatrial septum of $>10$ $\mathrm{mm}$ from the plane of atrial septum into either right or left atria or a combined total excursion into either atrial chamber of $>15 \mathrm{~mm}$. In general population, $83 \%$ of patients with ASA have concomitant presence of $\mathrm{PFO}$ as diagnosed on TEE. ${ }^{2}$ ASA have been associated with the size of a PFO, multiple septal fenestrations, and increased risk of cryptogenic stroke or embolic events. The risk of cryptogenic stroke tends to exponentially increase in the presence of $\mathrm{PFO}$ (odds ratio [OR], 1.83; 95\% confidence interval [CI], 1.25-2.66), ASA (OR, 2.35; 95\% CI, 1.46-3.77), and combined PFO with ASA (OR, 4.96; 95\% CI, 2.37-10.39). ${ }^{13}$ Various mechanisms have been proposed for additional increased risk in cryptogenic stroke due to the presence of ASA with PFO: risk for thrombus formation due to turbulent flow across a PFO tunnel or perianeurysmal pockets or local flow stasis or via incomplete atrial emptying, ${ }^{14}$ or hemodynamic allowance of paradoxical emboli via the $\mathrm{PFO}$ conduit. ${ }^{15}$

\section{PFO characteristics}

Various specific characteristics of a PFO that need detailed assessment during management of cryptogenic stroke patients include size, associated causality risk, and volume of right-to-left flow across a PFO. PFOs have a mean diameter of $4.9 \mathrm{~mm}$ (range, 1-19) sufficient enough to allow passage of paradoxical emboli through the shunt and occlude the middle cerebral artery stem ( $3 \mathrm{~mm}$ ) or distal cortical branches $(1 \mathrm{~mm}){ }^{2}$ Approximately 98\% of PFOs fall in the size range of $1-10 \mathrm{~mm}$ based on an autopsy-based study. ${ }^{16}$ Apart from the actual size of a PFO (i.e., maximum separation of the septum primum from the secundum), another working definition of PFO is commonly used based on the number of microbubbles detected in either left atrium or cerebral blood vessels. A large shunt size refers to the detection of $>20$ microbubbles in the left atrium within 3 cardiac cycles after opacification of the right atrium..$^{17}$ Although, definition for a high-risk PFO varies in the published literature, majority of studies have defined it as either concomitant presence of ASA, hypermobility of atrial septum (combined excursion into either atrium $\geq 10$ $\mathrm{mm}$ ), or a PFO size $\geq 2 \mathrm{~mm}$ in height and $\geq 10 \mathrm{~mm}$ in length. ${ }^{11,18}$

\section{Increased RLS flow across PFO}

Blood flow across the PFO additionally determines the risk of cryptogenic stroke. With a greater volume of blood flow across the RLS via PFO, there is an increased 
chance of paradoxical emboli to pass directly through the PFO conduit rather flow in to the right ventricle. Increased RLS likely occurs in patients with a large PFO size and chronic pulmonary hypertension that fosters paradoxical flow in cardiac chambers. However, transient increase in RLS during VM -forceful expiration against a closed glottis- occurs mainly at phase III of this maneuver due to the increase in blood flow towards right heart chambers. Few other daily activities that momentarily increase the thoracic pressure include heavy weight lifting, straining at stool, sexual intercourse, coughing, sneezing, and vomiting. ${ }^{19}$ Interestingly, few studies have demonstrated stroke location and pattern to differentiate between a constant and provoked RLS. Patients with VM provoked RLS were observed to have predominance of posterior circulation infarcts, suggesting VM likely promote RLS and paradoxical embolism to vertebrobasilar circulation..$^{20}$

Similarly, forceful inspiration against a closed glottis (Muller maneuver) tends to momentarily reduce the thoracic pressure and additionally have been associated with an increase in RLS. ${ }^{21}$ Muller maneuver occurs as vigorous inspiratory efforts during apneic episodes that triggers stroke onset during sleep in patients with a history of obstructive sleep apnea. Moreover, obstructive sleep apnea is associated with pulmonary hypertension that further tends to increase the RLS across PFO. Accordingly, a thorough clinical history remains paramount to correlate symptom onset that coincides with above-mentioned activities that lends support to PFO causality.

\section{Imaging pattern for paradoxical embolism}

Based on specific diffusion-weighted image lesion pattern observed on magnetic resonance imaging (MRI), stroke mechanisms can be ascertained to further differentiate various etiopathogenic mechanisms. ${ }^{22}$ Prior collaborative database study suggested large, radiologically apparent and superficially located infarcts as more likely to be PFO-related than cryptogenic strokes of other causes. ${ }^{23}$ Similarly, a MRI based study have demonstrated PFO-related strokes present as single cortical lesion or multiple small scattered lesions, and are more often located in the vertebrobasilar circulation. ${ }^{24}$

Additionally, paradoxical emboli present with unique clinical characteristics. Prevalence of venous thrombosis among cryptogenic stroke patients varies considerably $(7.2-40 \%)$ and is highly dependent on the type of imaging modality. ${ }^{25}$ Although noninvasive testing for paradoxical embolism include lower extremity doppler ultrasound that detects superficial or deep venous thrombi, the study is impeded by limited sensitivity and reliance on the skills of ultrasound technician. ${ }^{26}$ Venous thrombi -formed in thrombogenic conditionsprecedes a paradoxical embolism via $\mathrm{PFO}$ and results in small distal infarcts without any visible large vessel occlusion on angiography. Clots formed either in venous system or during turbulent flow within long PFO tunnel often tend to be firm rendering a lower recanalization rate in comparison to fresh clots by atrial fibrillation.

\section{DIAGNOSTIC MODALITIES FOR MANAGEMENT OF PFO}

The evaluation of PFO relies on a detailed assessment with the use of various diagnostic modalities. In the following section, we discuss various available diagnostic modalities that are routinely used for detailed assessment of cardio-aortic anomalies.

\section{Transthoracic echocardiography (TTE)}

TTE is the most widely and commonly used ultrasound modality to evaluate interatrial septum. It usually involves the use of an agitated saline contrast to identify the presence or absence of a RLS and henceforth, an existence of PFO. Although TTE remains as the preferred initial diagnostic modality, the poor image quality does not permit a detailed cardiac evaluation. Moreover, TTE is least sensitive among all routinely used modalities to detect PFO and detects only one-half the instances of PFOs found either on TEE or TCD. Standardized protocols including multiple injections of agitated saline with VM need to be followed to increase the accuracy of echocardiographic procedures (Table 1). Therefore, after initial evaluation with TTE, TEE should be performed prior to consideration for PFO closure to further characterize the atrial septal abnormality and other related risk factors. ${ }^{1}$ 
TABLE 1. Standardized protocol for echocardiography to increase accuracy of PFO detection

Intravenous catheter is typically placed in antecubital vein

Using a 10-mL syringe connected to a three-way locking stopcock, combine $1 \mathrm{~mL}$ of air with 1-mL of patient's blood and 8-mL of sa-

line (9-mL saline if avoiding use of blood)

Addition of blood increases the rate of microbubble detection using echocardiography

Rapid back and forth mixture of contrast solution into an empty 10-mL syringe attached to the stopcock produces agitated bubbles

The agitated saline contrast is exchanged between 10- $\mathrm{mL}$ syringe at least 10 times and injected as a bolus during normal respiration (rest)

Repeat the testing using VM: Inject agitated contrast 5 seconds before starting VM while maintaining VM for at least 10 seconds.

Rapidly inject the agitated bubbles through antecubital vein while acquiring a long clip length (i.e., 10 seconds) with the echocardiography system

Record echocardiographic images from the four-chamber view for TTE, and the angle best profiling the atrial septum is used for TEE, usually $30-120^{\circ}$

Use of biplane imaging on TEE might enhance detection of a small RLS

PFO; patent foramen ovale, VM; valsalva maneuver, TTE; transthoracic echocardiography, TEE; transesophageal echocardiography, RLS; right-to-left shunt.

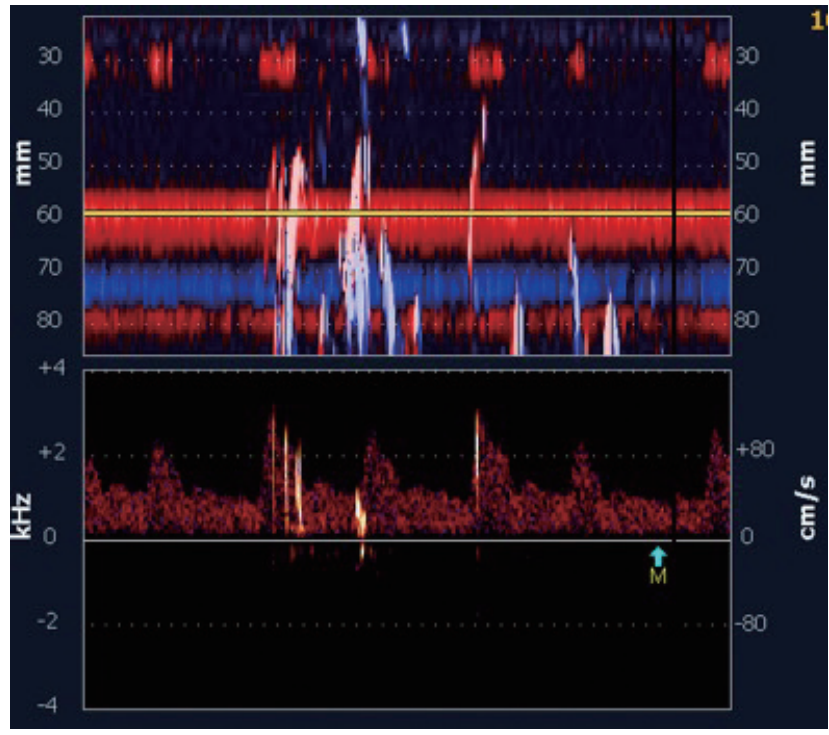

FIG. 1. Transcranial Doppler monitoring to assess the grading for right-to-left shunt.

\section{TCD ultrasonography}

TCD is an established, cost-effective, and a valid imaging modality for PFO detection. Using pulsed-wave Doppler ultrasonography, it involves intracranial detection of microbubbles that have crossed from venous to arterial circulation across the PFO tunnel (Fig. 1). Routinely, the contrast agent is prepared mixing $9 \mathrm{~mL}$ of isotonic saline solution and $1 \mathrm{~mL}$ of air with a threeway stopcock to prepare a mixture of agitated saline and is injected intravenously during different phases of respiration (at rest and during VM). To enhance the detection rate of microbubbles, $1 \mathrm{~mL}$ of patient's blood is mixed with $1 \mathrm{~mL}$ air and $8 \mathrm{~mL}$ of isotonic saline. In comparison to TTE or TEE, it has advantages of increased patient comfort, non-invasive technique (compared with TEE), rapid and semiquantitative assessment of shunt size, and has an ability to identify extracardiac and intracardiac shunting. Similar to TTE and TEE, TCD studies can be performed in normal respiration and VM to maximize the sensitivity and specificity for detection of RLS. TCD can detect $90-100 \%$ of PFOs found on TEE and up to $10 \%$ of PFOs that are missed on TEE. ${ }^{2}$ However, it lacks in providing additional information on other structural cardiac lesions and aortic-arch atherosclerosis.

Apart from quantifying the cerebral burden of paradoxical embolism, TCD monitoring involves detection of spontaneous micro embolic signals (MES). Prior studies have demonstrated a mean latency in injection-to-detection of microbubbles in middle cerebral artery of <11 seconds (latency limit of PFO detection in TCD), while greater latency periods (especially $>30$ seconds) suggested extracardiac shunts or pulmonary fistula. ${ }^{27}$ To quantify and standardize the MES detection using TCD, two grading systems are commonly used. The International Consensus Criteria involves unilateral monitoring of MES using a single-gate TCD that comprises of a four-level categorization ranging from an absence of MES (negative result) to a 'curtain pattern' (too numerous MES to count) ${ }^{28}$ (Fig. 2). Whereas, Spencer's Logarithmic Scale (SLS), six-point logarithmic scale, is a more commonly used grading scale in which higher grades refer to a larger RLS. ${ }^{29}$ 


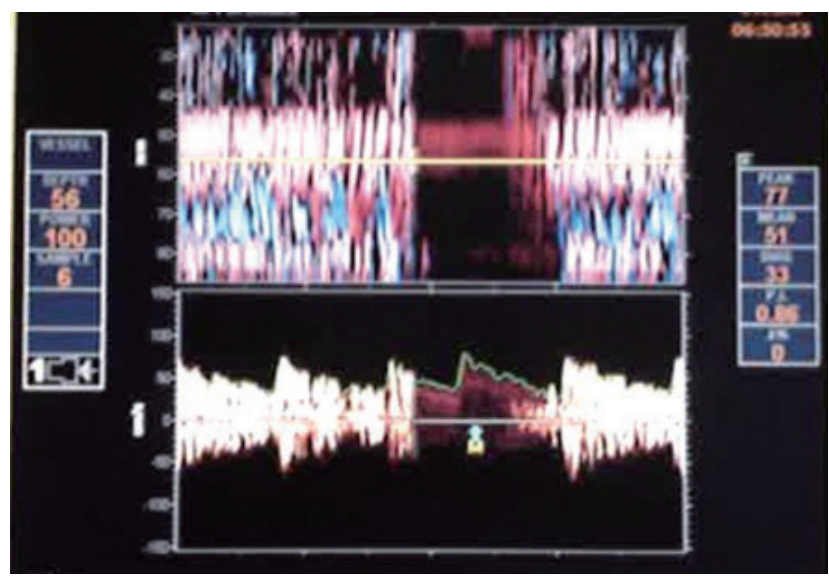

Fig. 2. Transcranial doppler monitoring demonstrating a large rightto-left shunt.

\section{TEE}

TEE remains as the gold standard diagnostic modality for detection of PFO (Supplementary Video 1). As compared to tissue confirmatory diagnosis on autopsy, TEE has a sensitivity of $\sim 90 \%$ and a specificity $>95 \%$. Previous studies have suggested that PFOs with the highest stroke risk on TEE have following characteristics: size $\geq 2 \mathrm{~mm}$, detection of $>20$ microbubbles in left atrium, RLS observed in resting state of respiration, and interatrial septum mobility of $>6.5 \mathrm{~mm} .^{27}$ TEE is a semi-invasive procedure, and if tolerated by the patients, uniquely characterizes PFO anatomy, presence of an ASA, margins or rims of the atrial septal defect (ASD) and the presence of additional proximal embolic etiologies such as aortic arch atheroma and atrial appendage thrombi. ${ }^{1}$ Additional provocative maneuvers such as VM should be performed to transiently increase right atrial pressure to further assist in PFO detection, however remains a challenge in sedated patients (Supplementary Video 2). The timing of bubble appearance in the left atrium is crucial in making the correct diagnosis and differentiate intracardiac from transpulmonary shunts. In case of a shunt at cardiac level such as PFO, microbubbles tend to appear in the left atrium within 3-6 cardiac beats after opacification of the right atrium (Fig. 3). Whereas the appearance of microbubbles in the left atrium after 3-6 beats indicate an intrapulmonary shunt, such as arteriovenous malformation. ${ }^{1}$

In contrast to routine TTE, both $2 \mathrm{D}$ and $3 \mathrm{D}$ TEE offers significant anatomic details and should be per-

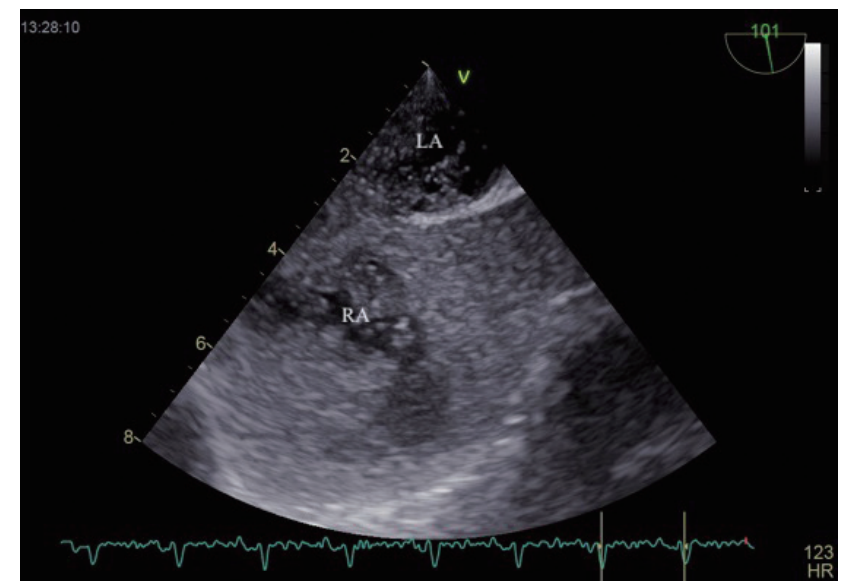

FIG. 3. Transesophageal echocardiography during Valsalva maneuver showing complete opacification of left atrium. RA; right atrium, LA; left atrium.

formed in all adult patients being evaluated for PFO closure. Specialized views involving slow sweep from $30-120^{\circ}$ provides an angle suited best for profiling the atrial septum. Various interatrial structural abnormalities such as ASD do not exist in a true flat plane, and thus $3 \mathrm{D}$ TEE is extremely helpful in these scenarios. In comparison to $2 \mathrm{D} \mathrm{TEE}$, real-time $3 \mathrm{D}$ TEE is more frequently used to better define $\mathrm{PFO}$ variations ${ }^{30}$ and procedural guidance for $\mathrm{PFO}$ closure as it may provide enface views of the atrial septum showing the relationship of the PFO and device with the surrounding structures. ${ }^{31}$

\section{Coronary computed tomographic angiography (CCTA)}

CCTA is a non-invasive imaging technique which is increasingly utilized to identify and/or confirm the potential sources of cardio-aortic sources of cerebral embolism. Previous studies have demonstrated CCTA as a useful diagnostic modality to detect high-risk cardiac sources of embolism in stroke patients. ${ }^{32}$ CCTA can provide direct and detailed visualization of atheromatous plaques of the aorta and other paradoxical embolic sources..$^{33}$ A Korean retrospective study compared the diagnostic feasibility and accuracy of the multidetector CCTA with TEE for detection of PFO. ${ }^{34}$ The authors concluded that a contrast agent jet from the left atrium towards right atrium toward the inferior vena cava in multiphase reconstruction CT images with channel-like appearance of the interatrial septum on CCTA images confirms the presence of a PFO. Furthermore, 
CCTA tends to detect smaller atheroma and likely is better in defining the morphology of atheromatous plaque. ${ }^{35}$ Although these technical advantages suggest a complimentary value of CCTA and TEE, ${ }^{36}$ CCTA involves administration of iodinated contrast dye and ionizing radiation that poses further limitations for its generalizability.

\section{ASSESSMENT OF DIAGNOSTIC MODALITIES}

\section{Comparative yields in PFO detection}

1) TCD vs. TTE

Multiple analyses have compared TTE and TCD to assess the diagnostic superiority between the two modalities for PFO detection. A systematic review and diagnostic test accuracy meta-analysis compared the diagnostic value of TTE with TCD. ${ }^{37}$ The authors included 35 studies that provided the sensitivity and specificity of TTE, TCD or both techniques in comparison to TEE (the gold standard). The authors reported a pooled sensitivity and specificity for TCD as $96.1 \%$ and 92.4\% whereas respective measures for TTE were $45.1 \%$ and $99.6 \%$, respectively. TTE was observed to be superior in regard to higher positive likelihood ratio values than TCD (suggesting a positive TTE finding is reliable in ruling in a PFO diagnosis), whereas TCD was associated with a lower negative likelihood values than TTE (suggesting negative TCD test is accurate in ruling out a PFO). Finally, the area under the summary receiver operating curve (AUC) was significantly greater for TCD (AUC, 0.98; 95\% CI, 0.97-0.99) as compared to TTE studies (AUC, 0.86; 95\% CI, 0.82-0.89). Their analyses add to the growing evidence that TCD is more sensitive but less specific as compared to TTE for PFO detection in patients with cryptogenic cerebral ischemia, and the overall diagnostic yield of TCD appears to outweigh that of TTE.

\section{2) TCD vs. TEE}

Prior studies have evaluated the concordance rates for RLS quantification between TCD and TEE diagnostic modalities. A prospective study analyzed the PFO detection and concordance rates while simultaneous performance of both the modalities at rest and with
VM states of respiration. ${ }^{27}$ They observed an almost perfect concordance in PFO detection and RLS quantification (Cohen's K=0.93). Similarly, another prospective study assessed the safety of TCD-bubble study and compared detection of RLS using TCD and TEE. ${ }^{38}$ TCD identified presence of RLS in 151/508 (30\%) cryptogenic cerebral ischemia patients. Of those, 101 patients were additionally tested for confirmation using TEE-bubble study. Similar to previous study, the authors observed detection of RLS in 99/101 patients that correlates to an agreement rate of $98 \%$ with TCD.

Moreover, TCD has been shown to have a better RLS detection rate in comparison to TEE. A Canadian study compared 284 patients with cryptogenic stroke and assessed RLS detection rates using both TCD and TEE. ${ }^{39}$ Among $15 \%$ of patients, TEE failed to detect RLS that was positively detected using TCD. Interestingly, $6 \%$ of those RLS were diagnosed as high grade ( $\geq$ grade 3) using SLS grading system for TCD monitoring. In comparison to TEE, the authors concluded that TCD is more sensitive for RLS detection and is a valuable technique for prediction of recurrent cerebral ischemic events and management of paradoxical embolism. Accordingly, various practice standards have recommended for TCD as having an equal or superior sensitivity for RLS detection in comparison to TTE or TEE. ${ }^{4 \circ}$ However, it should be noted that TCD depends on a mean latency in injection-to-detection period to differentiate RLS of cardiac from RLS of non-cardiac origin, and thus TEE is more accurate than TCD to detect the cardiac origin of RLS via PFO.

\section{Technical advantages and disadvantages}

TTE is the most commonly used and is still the preferred diagnostic modality worldwide for initial cardiac investigation for patients with cerebrovascular ischemic events. It provides visualization of cardiac structures and help in diagnosing co-existing ASA and other cardiac pathologies. However due to poor image quality and sensitivity for RLS detection, ${ }^{41}$ TTE is being lately considered as an insufficient screening tool in patients with ischemic stroke.

TEE remains as gold standard and provides real-time images of cardiac pathologies including PFO and ASA, but can also diagnose aortic pathologies and other 
cardiac entities that can cause stroke (e.g., cardiac mases-myxoma or endocarditis). It can additionally detect thrombus presence within a PFO tunnel and is currently an indispensable procedure prior (and during) the consideration for PFO closure. However, TEE is a semi-invasive diagnostic technique that requires frequent reliance over experienced echocardiographers. The procedure usually requires sedation and can be uncomfortable for ischemic stroke patients with residual aphasia or dysphagia. Moreover, TEE commonly renders a suboptimal VM for PFO detection (Table 2). Although the procedure is now frequently performed at bedside of the patient, no food intake by mouth overnight with potential scheduling delays are few disadvantages that tend to prolong the hospitalization.

On the contrary, TCD is a non-invasive, cost-effective, fast, valid, and easily reproducible bedside procedure that uses a pulsed-wave Doppler ultrasonography. ${ }^{42,43}$ It provides optimal calibrated VM (Supplementary Video 3) and detects small PFOs that can potentially be missed by TEE. Additionally, TCD does not require sedation that poses a challenge in neurological exam in acute stroke patients. In comparison to an effective VM during TCD, it can be a technical challenge to perform VM during TEE, especially in elderly patients with severe neurological deficits.

Several methodological improvements have been suggested to further increase the sensitivity of TCD: performing the procedure twice in a supine position, using a $25-40$ second time window to exclude other sources of shunting, using bilateral trans-temporal recordings, performing VM for 10 seconds after $5-10$ seconds of contrast injection, using alternative positions (right lateral decubitus, upright sitting, and sitting

TABLE 2. Technical challenges with TEE for PFO detection leading to false positive and false negative findings

\begin{tabular}{|c|c|}
\hline False positive findings & False negative findings \\
\hline 1. Intrapulmonary shunts (such as arteriovenous malformation) & 1. Inadequate RA opacification \\
\hline 2. Inter-atrial septal defects (such as ASD) & 2. Inadequate VM \\
\hline \multirow[t]{3}{*}{$\begin{array}{l}\text { 3. Pseudo-contrast; caused by the strain phase of } \mathrm{VM} \text { with tran- } \\
\text { sient stagnation of blood in the pulmonary veins }\end{array}$} & $\begin{array}{l}\text { 3. Poor imaging quality (due to obesity, lack of cooperation, or seda- } \\
\text { tion) }\end{array}$ \\
\hline & $\begin{array}{l}\text { 4. Eustachian valve directing venous return from the IVC to the atrial } \\
\text { septum (preventing microbubbles entering from the SVC to cross } \\
\text { the atrial septum) }\end{array}$ \\
\hline & $\begin{array}{l}\text { 5. Inability to increase the RA pressure above the LA pressure (LV } \\
\text { diastolic dysfunction) }\end{array}$ \\
\hline
\end{tabular}

TEE; transesophageal echocardiography, PFO; patent foramen ovale, RA; right atrium, ASD; atrial septal defect, VM; valsalva maneuver, IVC; inferior vena cava, SVC; superior vena cava, LA; left atrium, LV; left ventricle.

TABLE 3. Technical consideration with TCD for PFO detection

Similar to standardized protocol for echocardiography, prepare agitated saline and inject bubbles via antecubital vein during normal respiration and $\mathrm{VM}$

At least one proximal MCA is insonated at ICA terminus (obtain MCA and/or ACA waveform if possible)

Register the time of appearance for initial bubbles at MCA level to differentiate intracardiac vs. extracardiac shunts

Perform the procedure twice in a supine position

Use a $25-40$ seconds time window to exclude other sources of shunt

Use bilateral trans-temporal recordings

Perform VM for 10 seconds at $5-10$ seconds after contrast injection

Alternate positions during TCD (right lateral decubitus, upright sitting, and sitting with right lateral leaning)

Prefer use of femoral vein instead of antecubital vein

Use patient's blood and increase the number of mixes

TCD; transcaranial doppler, PFO; patent foramen ovale, VM; valsalva maneuver, MCA; middle cerebral artery, ICA; internal carotid artery, ACA; anterior cerebral artery. 
with right lateral leaning) ${ }^{44}$ using femoral instead of antecubital vein, ${ }^{45}$ and increasing the number of mixes especially with patient's blood (Table 3) ${ }^{43,46}$ However, TCD involves inherent limitations as it is operator dependent and does not visualize PFO, ASA, cardio-aortic atherosclerosis or other cardiac pathologies. Although an alternative trans-occipital insonation of posterior circulation tends to minimize screening failure, ${ }^{47} \mathrm{ab}-$ sence of trans-temporal windows in $10-15 \%$ of patients limits its usage in patients older than 60 years of age..$^{8}$

Both TCD and TEE provide detection of PFO and quantification of shunt size. TEE is considered the gold standard in the evaluation of cryptogenic stroke, whereas its frequent use is limited among patients with acute stroke due to mental status change, coagulopathy/ bleeding tendency, and/or lack of patient cooperation. On the contrary, TCD has equal to superior sensitivity for PFO detection, and thus the two techniques are therefore complementary. ${ }^{27}$

\section{RELEVANCE OF PFO AND UPDATED RECOM- MENDATIONS FOR MANAGEMENT}

The diagnostic workup in a patient with cryptogenic stroke remains of paramount importance and requires a thorough initial diagnostic workup. All young and middle-aged patients diagnosed with cerebral ischemia should undergo detailed evaluation to identify atherosclerosis in large or small cerebral arteries, nonatherosclerotic arteriopathies, structural and dysrhythmic cardio-aortic sources of embolism, and hypercoagulable states ${ }^{3}$ before branding a diagnosis of 'cryptogenic stroke'. Additionally, presence of RLS pose challenges whether the PFO or other structural causes for RLS are an incidental finding or causative for the index event. To assess the causality associated with a detected PFO, a risk of paradoxical embolism (RoPE) score was developed using data from 12 component studies. ${ }^{4}$ Patients with high RoPE scores (younger age, no vascular risk factors, and imaging showing cortical infarct) are more likely to have pathogenic PFOs, whereas low RoPE scores (older age group with vascular risk factors) suggested that a detected PFO is probably incidental. Additionally, presence of high-grade alternative etiologies tends to greatly reduce the PFO-attributable fraction as
PFO carries a status of being a medium-grade etiology for embolism. ${ }^{49}$

Treatment options available for secondary stroke prevention in patients with cryptogenic ischemic stroke and PFO include antiplatelet therapy (APT), oral anticoagulation (OAC), and percutaneous PFO closure. Until recently, clinicians preferred use of APT alone more often in comparison to OAC. Two RCTs - PFO in Cryptogenic Stroke Study (PICSS) ${ }^{50}$ and Patent Foramen Ovale Closure or Anticoagulants versus Antiplatelet Therapy to Prevent Stroke Recurrence (CLOSE), ${ }^{10}$ evaluated the efficacy between the two approaches among the subgroup of patients with cryptogenic ischemic stroke and PFO. There was no difference in the efficacy in either PICSS (hazard ratio [HR], 0.52; 95\% CI, 0.16-1.67) $)^{50}$ or CLOSE (HR, 0.44; 95\% CI, 0.11-1.48) ${ }^{10}$ trials. Similarly, a prior individual participant data meta-analysis involving 12 prospective observational studies demonstrated no significant difference (HR, 0.75; 95\% CI, 0.44-1.27) between the efficacy of APT and OAC. ${ }^{51}$ Recently conducted subgroup analysis from the NAVIGATE ESUS trial also demonstrated no difference (HR, 0.54; 95\% CI, 0.22-1.36) between OAC and APT for stroke recurrence among cryptogenic stroke patients with $\mathrm{PFO} .{ }^{2}$ Interestingly, the authors pooled their data with two previous RCTs (CLOSE and PICSS), and demonstrated a favorable efficacy for OAC (OR, 0.48; 95\% CI, 0.24-0.96) without any heterogeneity. Furthermore, a recent network meta-analysis involving data from 6 RCTs demonstrated a significant benefit with OAC (vs. APT) in recurrent stroke prevention (OR, 0.42; 95\% CI, 0.22-0.78).53 However, in comparison to bleeding risk associated with APT, previous RCTs ${ }^{10,50}$ and meta-analyses ${ }^{53,54}$ have consistently demonstrated a significant increase in bleeding risk among patients treated with OAC. Accordingly, US national practice guidelines ${ }^{55,56}$ have previously endorsed APT whereas recent European guidelines ${ }^{54}$ have provided a conditional statement for either OAC or APT as a choice based on individual safety and risk of stroke recurrence. In comparison to vitamin $\mathrm{K}$ antagonist, non-vitamin-K antagonist oral anticoagulants provide promising alternative with a comparable efficacy in DVT prevention and a lower bleeding risk. ${ }^{52,57,58}$ Future studies are needed to compare their efficacy in large prospective cohorts of PFO patients.

Until recently, no randomized data was available to 
show a convincing benefit for PFO closure as compared to medical therapy alone. Accordingly, current recommendations from the American Heart Association, American Stroke Association, and American Academy of Neurology report no benefit for PFO closure compared to medical treatment (class III; level of evidence A). ${ }^{55,56}$ However, 3 RCTs in $2017^{8-10}$ and another in $2018^{11}$ reaffirmed the longstanding debate on the efficacy of PFO closure in comparison to medical therapy alone. The data demonstrated a significant reduction in stroke recurrence among those patients that were assigned to PFO closure combined with APT as compared to those assigned to APT alone. Subsequently, multiple network meta-analyses confirmed the superior efficacy of $\mathrm{PFO}$ closure plus APT (8.7\% absolute risk reduction over 5 years) in comparison to APT alone. ${ }^{59,60}$ However, PFO closure probably incurs persistent atrial fibrillation (AF) (1.8\% absolute risk increase) and procedural complications (3.6\% absolute risk) during first year after the procedure. ${ }^{43,59}$ As PFO may serve as a pop-off valve in case of increased left atrial pressure, PFO closure may further contribute to increased left atrial pressure and AF. Recent meta-analysis additionally assessed various PFO closure devices vs medical therapy for efficacy and safety outcomes. In regard to the risk of ischemic stroke or TIA (vs. risk of new-onset AF), Amplatzer device was demonstrated as the optimal therapeutic option, whereas STARFlex was the worst option among all the available devices. ${ }^{60}$

PFO closure for cryptogenic strokes in a highly selective patient cohort is cost-effective, produces benefit in quality adjusted life years gained and cumulates to potential cost savings over following years. ${ }^{61}$ However, patient selection remains crucial as even marginal declines in the treatment effectiveness dramatically reduce the potential cost benefit. Accordingly, recent clinical practice recommendations ${ }^{62}$ and European practice guidelines ${ }^{54}$ have strongly endorsed in favor of PFO closure for carefully selected patients aged 18-65 years with a confirmed diagnosis of cryptogenic stroke, TIA or systemic embolism. Based on equivalent efficacy of PFO closure and OAC in a recent meta-analysis, ${ }^{53}$ the expert panel additionally made a weak recommendation in favor of PFO closure and APT (vs. OAC).

\section{CONCLUSION}

The diagnostic management in cryptogenic stroke requires a thorough initial workup and multi-team collaboration. Detection of PFO and establishing its causality as a source of paradoxical embolism remains crucial. Absence of vascular risk factors and posterior circulation involvement in neuroimaging are thought to be suggestive of PFO presence in patients with cryptogenic stroke. TTE is most commonly used initial diagnostic modality, however due to poor sensitivity, it should not be the sole diagnostic modality. TCD with superior sensitivity is recommended as a preferred initial screening test that complements TEE for further PFO confirmation. Different technical considerations of both TCD and TEE opens future horizons for further improved diagnostic yield in PFO detection. Recent emergence of randomized data has ushered a new enthusiasm, confirming PFO closure with a genuine benefit in carefully selected young and middle-aged patients with cryptogenic ischemic stroke. In comparison to medical management, practice guidelines have echoed strong recommendations for PFO closure followed by APT. Diagnostic workup, either TCD or TEE, should be performed within 6 months in patients who underwent PFO closure to detect device stability. Future management of cryptogenic stroke patients calls for collaborative efforts among neurologists and cardiologists for further reduction in stroke recurrence via detailed etiologic evaluations, exclude other causes of stroke, use detailed diagnostic modalities to identify high-risk patient features, and provide treatment recommendations to young and middle-aged ischemic stroke patients with PFO.

\section{SUPPLEMENTARY MATERIALS}

Supplementary materials related to this article can be found online at https://doi.org/10.31728/jnn.2019.00042.

\section{REFERENCES}

1. Silvestry FE, Cohen MS, Armsby LB, Burkule NJ, Fleishman CE, Hijazi ZM, et al. Guidelines for the echocardiographic 
assessment of atrial septal defect and patent foramen ovale: from the American Society of Echocardiography and Society for Cardiac Angiography and Interventions. J Am Soc Echocardiogr. 2015;28:910-958.

2. Saver JL, Mattle HP, Thaler D. Patent foramen ovale closure versus medical therapy for cryptogenic ischemic stroke: a topical review. Stroke. 2018;49:1541-1548.

3. Saver JL. Clinical practice. Cryptogenic stroke. N Engl J Med. 2016;374:2065-2074.

4. Kent DM, Ruthazer R, Weimar C, Mas JL, Serena J, Homma $S$, et al. An index to identify stroke-related vs incidental patent foramen ovale in cryptogenic stroke. Neurology. 2013;81:619-625.

5. Meier B, Kalesan B, Mattle HP, Khattab AA, Hildick-Smith D, Dudek D, et al. Percutaneous closure of patent foramen ovale in cryptogenic embolism. N Engl J Med. 2013;368:10831091.

6. Carroll JD, Saver JL, Thaler DE, Smalling RW, Berry S, MacDonald LA, et al. Closure of patent foramen ovale versus medical therapy after cryptogenic stroke. $N$ Engl J Med. 2013;368:1092-1100.

7. Furlan AJ, Reisman M, Massaro J, Mauri L, Adams H, Albers $\mathrm{GW}$, et al. Closure or medical therapy for cryptogenic stroke with patent foramen ovale. N Engl J Med. 2012;366:991-999.

8. Saver JL, Carroll JD, Thaler DE, Smalling RW, MacDonald LA, Marks DS, et al. Long-term outcomes of patent foramen ovale closure or medical therapy after stroke. $\mathrm{N}$ Engl J Med. 2017;377:1022-1032.

9. Søndergaard L, Kasner SE, Rhodes JF, Andersen G, Iversen HK, Nielsen-Kudsk JE, et al. Patent foramen ovale closure or antiplatelet therapy for cryptogenic stroke. N Engl J Med. 2017;377:1033-1042.

10. Mas JL, Derumeaux G, Guillon B, Massardier E, Hosseini H, Mechtouff L, et al. Patent foramen ovale closure or anticoagulation vs. antiplatelets after stroke. $N$ Engl J Med. 2017;377:1011-1021.

11. Lee PH, Song JK, Kim JS, Heo R, Lee S, Kim DH, et al. Cryptogenic stroke and high-risk patent foramen ovale: the DEFENSE-PFO trial. J Am Coll Cardiol. 2018;71:2335-2342.

12. Alsheikh-Ali AA, Thaler DE, Kent DM. Patent foramen ovale in cryptogenic stroke: incidental or pathogenic? Stroke. 2009;40:2349-2355.

13. Leong MC, Uebing A, Gatzoulis MA. Percutaneous patent foramen ovale occlusion: current evidence and evolving clinical practice. Int J Cardiol. 2013;169:238-243.

14. Rigatelli G, Aggio S, Cardaioli P, Braggion G, Giordan M,
Dell'avvocata $\mathrm{F}$, et al. Left atrial dysfunction in patients with patent foramen ovale and atrial septal aneurysm: an alternative concurrent mechanism for arterial embolism? JACC Cardiovasc Interv. 2009;2:655-662.

15. Bushi D, Grad Y, Einav S, Yodfat O, Nishri B, Tanne D. Hemodynamic evaluation of embolic trajectory in an arterial bifurcation: an in-vitro experimental model. Stroke. 2005;36:2696-2700.

16. Hagen PT, Scholz DG, Edwards WD. Incidence and size of patent foramen ovale during the first 10 decades of life: an autopsy study of 965 normal hearts. Mayo Clin Proc. 1984;59:17-20.

17. Mazzucco S, Li L, Binney L, Rothwell PM; Oxford Vascular Study Phenotyped Cohort. Prevalence of patent foramen ovale in cryptogenic transient ischaemic attack and non-disabling stroke at older ages: a population-based study, systematic review, and meta-analysis. Lancet Neurol. 2018;17:609-617.

18. Takafuji H, Hosokawa S, Ogura R, Hiasa Y. Correction to: percutaneous transcatheter closure of high-risk patent foramen ovale in the elderly. Heart Vessels. 2019 Apr 15 [Epub]. https://doi.org/10.1007/s00380-019-01408-y.

19. Ozdemir AO, Tamayo A, Munoz C, Dias B, Spence JD. Cryptogenic stroke and patent foramen ovale: clinical clues to paradoxical embolism. J Neurol Sci. 2008;275:121-127.

20. Kim BJ, Kim NY, Kang DW, Kim JS, Kwon SU. Provoked right-to-left shunt in patent foramen ovale associates with ischemic stroke in posterior circulation. Stroke. 2014;45:37073710.

21. Mojadidi MK, Bokhoor PI, Gevorgyan R, Noureddin N, MacLellan WC, Wen E, et al. Sleep apnea in patients with and without a right-to-left shunt. J Clin Sleep Med. 2015;11:12991304 .

22. Malhotra K, Liebeskind DS. Overview of neuroimaging of stroke. In: Caplan LR, Biller J, Leary MC, Lo EH, Thomas AJ, Yenari M, et al. 2nd ed. Primer on Cerebrovascular Diseases. Cambridge: Elsevier. 2017;676-685.

23. Thaler DE, Ruthazer R, Di Angelantonio E, Di Tullio MR, Donovan JS, Elkind MS, et al. Neuroimaging findings in cryptogenic stroke patients with and without patent foramen ovale. Stroke. 2013;44:675-680.

24. Kim BJ, Sohn H, Sun BJ, Song JK, Kang DW, Kim JS, et al. Imaging characteristics of ischemic strokes related to patent foramen ovale. Stroke. 2013;44:3350-3356.

25. Lapergue B, Decroix JP, Evrard S, Wang A, Bendetowicz D, Offroy MA, et al. Diagnostic yield of venous thrombosis 
and pulmonary embolism by combined CT venography and pulmonary angiography in patients with cryptogenic stroke and patent foramen ovale. Eur Neurol. 2015;74:69-72.

26. Stöllberger C, Slany J, Schuster I, Leitner H, Winkler WB, Karnik R. The prevalence of deep venous thrombosis in patients with suspected paradoxical embolism. Ann Intern Med. 1993;119:461-465.

27. Belvís R, Leta RG, Martí-Fàbregas J, Cocho D, Carreras F, Pons-Lladó G, et al. Almost perfect concordance between simultaneous transcranial Doppler and transesophageal echocardiography in the quantification of right-to-left shunts. J Neuroimaging. 2006;16:133-138.

28. Jauss M, Zanette E. Detection of right-to-left shunt with ultrasound contrast agent and transcranial Doppler sonography. Cerebrovasc Dis. 2000;10:490-496.

29. Spencer MP, Moehring MA, Jesurum J, Gray WA, Olsen JV, Reisman M. Power m-mode transcranial Doppler for diagnosis of patent foramen ovale and assessing transcatheter closure. J Neuroimaging. 2004;14:342-349.

3o. Tanaka J, Izumo M, Fukuoka Y, Saitoh T, Harada K, Harada $\mathrm{K}$, et al. Comparison of two-dimensional versus real-time three-dimensional transesophageal echocardiography for evaluation of patent foramen ovale morphology. Am J Cardiol. 2013;111:1052-1056.

31. Rana BS, Shapiro LM, McCarthy KP, Ho SY. Three-dimensional imaging of the atrial septum and patent foramen ovale anatomy: defining the morphological phenotypes of patent foramen ovale. Eur J Echocardiogr. 2010;11:i19-i25.

32. Kim SJ, Choe YH, Park SJ, Kim GM, Chung CS, Lee KH, et al. Routine cardiac evaluation in patients with ischaemic stroke and absence of known atrial fibrillation or coronary heart disease: transthoracic echocardiography vs. multidetector cardiac computed tomography. Eur J Neurol. 2012;19:317-323.

33. Bang OY, Ovbiagele B, Kim JS. Evaluation of cryptogenic stroke with advanced diagnostic techniques. Stroke. 2014;45:1186-1194.

34. Kim YJ, Hur J, Shim CY, Lee HJ, Ha JW, Choe KO, et al. Patent foramen ovale: diagnosis with multidetector CT--comparison with transesophageal echocardiography. Radiology. 2009;250:61-67.

35. Hussain SI, Gilkeson RC, Suarez JI, Tarr R, Schluchter M, Landis DM, et al. Comparing multislice electrocardiogram-gated spiral computerized tomography and transesophageal echocardiography in evaluating aortic atheroma in patients with acute ischemic stroke. J Stroke Cerebrovasc
Dis. 2008;17:134-140.

36. Sipola P, Hedman M, Onatsu J, Turpeinen A, Halinen M, Jäkälä $\mathrm{P}$, et al. Computed tomography and echocardiography together reveal more high-risk findings than echocardiography alone in the diagnostics of stroke etiology. Cerebrovasc Dis. 2013;35:521-530.

37. Katsanos AH, Psaltopoulou T, Sergentanis TN, Frogoudaki A, Vrettou AR, Ikonomidis I, et al. Transcranial Doppler versus transthoracic echocardiography for the detection of patent foramen ovale in patients with cryptogenic cerebral ischemia: a systematic review and diagnostic test accuracy meta-analysis. Ann Neurol. 2016;79:625-635.

38. Tsivgoulis G, Stamboulis E, Sharma VK, Heliopoulos I, Voumvourakis K, Teoh HL, et al. Safety of transcranial Doppler 'bubble study' for identification of right to left shunts: an international multicentre study. J Neurol Neurosurg Psychiatry. 2011;82:1206-1208.

39. Tobe J, Bogiatzi C, Munoz C, Tamayo A, Spence JD. Transcranial Doppler is complementary to echocardiography for detection and risk stratification of patent foramen ovale. Can J Cardiol. 2016;32:986.

40. Alexandrov AV, Sloan MA, Tegeler CH, Newell DN, Lumsden A, Garami Z, et al. Practice standards for transcranial Doppler (TCD) ultrasound. Part II. Clinical indications and expected outcomes. J Neuroimaging. 2012;22:215-224.

41. Beattie JR, Cohen DJ, Manning WJ, Douglas PS. Role of routine transthoracic echocardiography in evaluation and management of stroke. J Intern Med. 1998;234:281-291.

42. Malhotra K, Conners JJ, Lee VH, Prabhakaran S. Relative changes in transcranial Doppler velocities are inferior to absolute thresholds in prediction of symptomatic vasospasm after subarachnoid hemorrhage. J Stroke Cerebrovasc Dis. 2014;23:31-36.

43. Sastry S, Daly K, Chengodu T, McCollum C. Is transcranial Doppler for the detection of venous-to-arterial circulation shunts reproducible? Cerebrovasc Dis. 2007;23:424-429.

44. Lao AY, Sharma VK, Tsivgoulis G, Malkoff MD, Alexandrov AV, Frey JL. Effect of body positioning during transcranial Doppler detection of right-to-left shunts. Eur J Neurol. 2007;14:1035-1039.

45. Gevorgyan R, Perlowski A, Shenoda M, Mojadidi MK, Agrawal H, Tobis JM. Sensitivity of brachial versus femoral vein injection of agitated saline to detect right-to-left shunts with transcranial Doppler. Catheter Cardiovasc Interv. 2014;84:992-996.

46. Gentile M, De Vito A, Azzini C, Tamborino C, Casetta I. 
Adding blood to agitated saline significantly improves detection of right-to-left shunt by contrast-transcranial color-coded duplex sonography. Ultrasound Med Biol. 2014;40:2637-2641.

47. Del Sette M, Dinia L, Rizzi D, Sugo A, Albano B, Gandolfo C. Diagnosis of right-to-left shunt with transcranial Doppler and vertebrobasilar recording. Stroke. 2007;38:2254-2256.

48. Alexandrov AV, Babikian VL, Adams RJ, Tegeler CH, Caplan LR, Spencer MP; National Stroke Association Panelists on Transcranial Doppler. The evolving role of transcranial Doppler in stroke prevention and treatment. J Stroke Cerebrovasc Dis. 1998;7:101-104.

49. Thaler DE, Ruthazer R, Weimar C, Mas JL, Serena J, Di Angelantonio E, et al. Recurrent stroke predictors differ in medically treated patients with pathogenic vs. other PFOs. Neurology. 2014;83:221-226.

50. Homma S, Sacco RL, Di Tullio MR, Sciacca RR, Mohr JP; PFO in Cryptogenic Stroke Study (PICSS) Investigators. Effect of medical treatment in stroke patients with patent foramen ovale: patent foramen ovale in cryptogenic stroke study. Circulation. 2002;105:2625-2631.

51. Kent DM, Dahabreh IJ, Ruthazer R, Furlan AJ, Weimar C, Serena J, et al. Anticoagulant vs. antiplatelet therapy in patients with cryptogenic stroke and patent foramen ovale: an individual participant data meta-analysis. Eur Heart J. 2015;36:2381-2389.

52. Kasner SE, Swaminathan B, Lavados P, Sharma M, Muir K, Veltkamp R, et al. Rivaroxaban or aspirin for patent foramen ovale and embolic stroke of undetermined source: a prespecified subgroup analysis from the NAVIGATE ESUS trial. Lancet Neurol. 2018;17:1053-1060.

53. Saber H, Palla M, Kazemlou S, Azarpazhooh MR, Seraji-Bozorgzad N, Behrouz R. Network meta-analysis of patent foramen ovale management strategies in cryptogenic stroke. Neurology. 2018;91:e1-e7.

54. Pristipino C, Sievert H, D’Ascenzo F, Louis Mas J, Meier B, Scacciatella P, et al. European position paper on the management of patients with patent foramen ovale. General approach and left circulation thromboembolism. Eur Heart J.
2018 Oct 25 [Epub]. https://doi.org/10.1093/eurheartj/ehy649.

55. Messé SR, Gronseth G, Kent DM, Kizer JR, Homma S, Rosterman L, et al. Practice advisory: recurrent stroke with patent foramen ovale (update of practice parameter): report of the guideline development, dissemination, and implementation Subcommittee of the American Academy of Neurology. Neurology. 2016;87:815-821.

56. Kernan WN, Ovbiagele B, Black HR, Bravata DM, Chimowitz MI, Ezekowitz MD, et al. Guidelines for the prevention of stroke in patients with stroke and transient ischemic attack: a guideline for healthcare professionals from the American Heart Association/American Stroke Association. Stroke. 2014;45:2160-2236.

57. Katsanos AH, Mavridis D, Parissis J, Deftereos S, Frogoudaki A, Vrettou AR, et al. Novel oral anticoagulants for the secondary prevention of cerebral ischemia: a network meta-analysis. Ther Adv Neurol Disord. 2016;9:359-368.

58. Malhotra K, Khunger M, Liebeskind DS. Non-vitamin K oral anticoagulants in stroke patients: practical issues. $J$ Stroke. 2017;19:104-106.

59. Mir H, Siemieniuk RAC, Ge LC, Foroutan F, Fralick M, Syed T, et al. Patent foramen ovale closure, antiplatelet therapy or anticoagulation in patients with patent foramen ovale and cryptogenic stroke: a systematic review and network meta-analysis incorporating complementary external evidence. BMJ Open. 2018;8:e023761.

6o. Tsivgoulis G, Katsanos AH, Mavridis D, Frogoudaki A, Vrettou AR, Ikonomidis I, et al. Percutaneous patent foramen ovale closure for secondary stroke prevention: network meta-analysis. Neurology. 2018;91:e8-e18.

61. Leppert MH, Poisson SN, Carroll JD, Thaler DE, Kim CH, Orjuela KD, et al. Cost-effectiveness of patent foramen ovale closure versus medical therapy for secondary stroke prevention. Stroke. 2018;49:1443-1450.

62. Kuijpers T, Spencer FA, Siemieniuk RAC, Vandvik PO, Otto CM, Lytvyn L, et al. Patent foramen ovale closure, antiplatelet therapy or anticoagulation therapy alone for management of cryptogenic stroke? A clinical practice guideline. Bmj. 2018;362:k2515. 